Relations industrielles

Industrial Relations

\title{
The Engineer in the Industrial Corporation, by Richard R. Ritti, New York, Columbia University Press, 1971, 261 pp.
}

\section{Laurent Bélanger}

Volume 28, numéro 3, 1973

URI : https://id.erudit.org/iderudit/028440ar

DOI : https://doi.org/10.7202/028440ar

Aller au sommaire du numéro

Éditeur(s)

Département des relations industrielles de l'Université Laval

ISSN

0034-379X (imprimé)

1703-8138 (numérique)

Découvrir la revue

Citer ce compte rendu

Bélanger, L. (1973). Compte rendu de [The Engineer in the Industrial Corporation, by Richard R. Ritti, New York, Columbia University Press, 1971, 261 pp.] Relations industrielles / Industrial Relations, 28(3), 677-678.

https://doi.org/10.7202/028440ar

Tous droits réservés @ C Département des relations industrielles de l'Université Laval, 1973
Ce document est protégé par la loi sur le droit d'auteur. L’utilisation des services d’Érudit (y compris la reproduction) est assujettie à sa politique d'utilisation que vous pouvez consulter en ligne.

https://apropos.erudit.org/fr/usagers/politique-dutilisation/ 
tions présentées ne découlent pas nécessairement et clairement de l'analyse.

Ce livre a cependant beaucoup plus de mérites et c'est ce qui en rend la lecture nécessaire à tous ceux qui s'intéressent au problème de la politique de main-d'oeuvre. D'abord, l'auteur met en lumière le problème vital des préoccupations de main-d'oeuvre dans une société industriclle avancée. Sur ce point, son message ressemble quelque peu à ce que Mangum et Levitan ont déjà avancé. En second lieu, Davidson dépeint très bien le fouillis administratif et organisationnel propre aux programmes américains de main-d'oeuvre. Il pose le problème d'une façon très intéressante. La citation suivante en est un exemple : « Program fragmentation is a product not only of agency rivalries but also, and more fundamentally, of the manner in which policies are formulated and sustained in a pluralist political system. As each new pressing need identified and publicized, a remedy or palliative is fashionned in the form of a governmental program. Thus government involvement tends to be a mosaic of singlepurpose efforts, with inevitable discard » (p. 7).

Ce premier chapitre dans lequel il présente le problème sous-étude est de loin le plus intéressant et le mieux fait du livre.

En troisième lieu, les préoccupations plus politiques de Davidson constituent le caractère original de ce travail. Cet aspect politique est en train de devenir, et avec raison, le sujet à la mode à l'intérieur des préoccupations de maind'oeuvre.

La plus grande leçon à tirer de ce livre par le lecteur non-américain réside sûrement dans l'apprentissage de ce qu'il ne faut pas faire pour organiser pratiquement des programmes de main-d'oeuvre. Les conflits entre niveaux de gouvernements, entre différents ministères et agences d'un même gouvernement et le fait que les préoccupations de main-d'oeuvre intéressent presque tout le monde exigent que la politique de main-d'oeuvre soit placée très haut dans la structure d'autorité. Espérer de la coordination entre plusieurs niveaux similaires d'autorité dans différents organismes peut être facilement une utopie. Cette coor- dination doit être forcée. C'est selon ce principe, sans que l'auteur le mentionne, qu'il en arrive à proposer une décentralisation sélective des programmes de main-d'oeuvre, décentralisation qui voit la définition des besoins et la livraison des services au niveau local, et la coordination, le financement, etc. à un niveau fédéral.

En résumé, ce livre présente des idées relativement récentes sur un sujet jusqu'ici très négligé mais crucial, à savoir la façon dont les services en main-d'oeuvre sont rendus. On sera sûrement appelé à réfléchir sur ce point bientôt au Canada et au Québec même si a priori plusieurs croient que de tels problèmes de coordination, de décentralisation et de «décatégorisation 》 ne se posent pas chez nous. Il sera alors sûrement utile de lire cet ouvrage de Davidson. En main-d'oeuvre, comme dans d'autres domaines, on a beaucoup à apprendre de l'expérience des autres.

\section{Jean SEXTON}

\section{Université Laval}

The Engineer in the Industrial Corporation, by Richard $R$. Ritti, New York, Columbia University Press, 1971, $261 \mathrm{pp}$.

On croit généralement que les laboratoires de recherche d'exploration et d'application constituent un repère où des individus hautement qualifiés peuvent encore exercer un certain contrôle dans l'organisation de leur travail et bénéficier ainsi de plus de latitude dans l'exercice de leurs fonctions. Cette croyance ne résiste pas complètement à l'observation des faits recueillis et analysés par Ritti dans douze laboratoires d'une grande entreprise américaine employant plus de 4,500 ingéneurs. Ritti, dans cet ouvrage, tente de dresser un profil de l'ingénieur malheureux sous deux aspects : celui de la sous-utilisation et du manque d'influence dans l'accomplissement de son rôle. Il recueille d'abord des données à l'échelle de l'entreprise et constate que la proportion des ingénieurs insatisfaits se rapproche de celle des techniciens et des ouvriers de la production. Il faut d'abord distinguer ces ingénieurs de ceux qui sont itinérants (field engineers) et des scientifiques qui présentent des profils spycho- 
logiques passablement différents. L'insatisfaction des ingénieurs de laboratoire se manifeste d'abord par une sous-utilisation de leur habileté et de leur potentiel. A la question que pensezvous de la somme de travail qu'on attend de vous?, les ingénieurs et les techniciens répondent qu'ils aimeraient en faire plus, alors que les autres catégories de personnel affirment que leur charge est trop lourde. Cette sous-utilisation peut-être attribuée à bien des facteurs dont le plus important demeure. celui de l'impossibilité d'arriver à une synchronisation parfaite des projets d'exploration et d'application. Une deuxième cause dans la conception que la hautedirection se fait de l'organisation du travail au sein d'un laboratoire. Cette conception s'apparente à un modèle d'organisation de type mécanique et bureaucratique qui transforme le laboratoire en une immense ligne de montage et d'assemblage qu'on retrouve dans les usines de fabrication de masse. L'insatisfaction se manifeste aussi par un manque d'influence dans la détermination de la mission technologique de chaque laboratoire. L'influence qu'on peut exercer dans ce domaine se trouve concentrée au sommet de la hiérarchie. Les décisions importantes sont centralisées à cet endroit parce que, semble-t-il, la coordination des projets et l'incertitude de leur durée l'exigent. De plus, ces décisions comportent des dimensions économiques, financières et organisationnelles dont l'ampleur échappe aux ingénieurs de la base ; du moins, c'est ce que la hautedirection prétend.

A travers ces deux manifestations et à l'aide de données recueillies sur les buts personnels des ingénieurs, l'auteur tente de cerner la cause fondamentale de l'insatisfaction. Elle réside dans un conflit entre les valeurs personnelles des individus et les exigences concrètes des tâches qu'on leur demande d'assumer. Contrairement à ce qu'on croit généralement, les valeurs et les buts personnels des ingénieurs sont en harmonie avec les objectifs de l'entreprise. Les ingénieurs, et c'est sur ce joint qu'ils diffèrent des scientifiques, épousent les valeurs patronales alors que les scientifiques souscrivent au schème de valeur des professions libérales.

Pour reprendre les termes de Gouldner, les ingénieurs seraient de mentalité «locale »; les scientifiques, de mentalité «cosmopolitan». Avec l'auteur, il faut reconnaître que le schème de référence qui met en opposition orientation professionnelle et orientation managériale ou bureaucratique ne peut expliquer l'insatisfaction des ingénieurs. Elle s'explique plutôt par l'impossibilité pour les ingénieurs de réaliser leurs buts personnels dans un contexte d'organisation du travail où la majorité des tâches sont fractionalisées et sont accomplies en suivant des spécifications rigides et détaillées. ILes ingénieurs veulent que l'entreprise soit à la fine pointe du progrès; ils veulent participer dans l'élaboration des objectifs des laboratoires, souhaitent une plus grande latitude dans l'organisation et le contrôle du travail du laboratoire. Par ailleurs, une organisation basée sur les principes $\overline{\mathrm{du}}$ taylorisme ne leur permet pas de satisfaire leurs aspirations. L'auteur fait suivre son analyse d'une liste de recommandations qui revêtent un caractère très général; il le fait en effectuant un examen critique des solutions déjà mises de l'avant en sciences du comportement, tels l'enrichissement des tâches, la revision des structures d'autorité, etc. Somme toute, c'est un ouvrage qui nous permet de mieux comprendre la situation de l'ingénieur en contexte industriel. Cependant, le volume n'est pas d'une lecture facile, puisqu'il n'obéit pas à un cadre de référence théorique initial qui permettrait de suivre le développement de la recherche, de localiser les variables importantes et de saisir les liens qu'elles entretiennent entre elles.

Laurent BELANGER 